\title{
Clinical, Biochemical and Molecular Profile of Variant Galactosemia in Children Detected by National Newborn Screening: A Pilot Study
}

\author{
Sylvia Capistrano-Estrada, ${ }^{1,2}$ Daffodil M. Canson ${ }^{1}$ and Catherine Lynn T. Silao ${ }^{1,2}$ \\ ${ }^{1}$ Institute of Human Genetics, National Institutes of Health, University of the Philippines Manila; \\ ${ }^{2}$ Department of Pediatrics, College of Medicine and Philippine General Hospital, University of the Philippines Manila
}

\begin{abstract}
Objective. The observed irregularities in the biochemical profile and the limited information on long-term outcomes among patients with Duarte variant (D/G) galactosemia have led to patient management variability. This study examined the molecular characteristics of Filipino patients with presumed variant galactosemia for confirmation of diagnosis. It also aimed to describe the corresponding biochemical, clinical and neurodevelopmental profiles in order to gain a better understanding of the patients with normal galactose metabolites in spite of low to absent GALT activity detected by the local newborn screening program.
\end{abstract}

Methods. Thirteen (13) patients who were presumed to have a variant form of galactosemia by national newborn screening between 2002 and 2010, and who previously underwent physical and neurodevelopmental assessment were included in the study. Repeat clinical, ophthalmologic and neurodevelopmental evaluations were done upon recruitment of participants. Direct sequence analysis of the coding region of the GALT gene was conducted to determine the patients' genotypes.

Results. None of the patients' genotypes were consistent with Duarte variant $(D / G)$ galactosemia. Their genotypes reflect the normal total blood galactose levels in patients, but were inconsistent with the absent or trace GALT activity.

Conclusion. Molecular testing for the entire cohort of presumed "variant" galactosemia Filipino patients will provide better profiling of this condition. Re-evaluation and assessment of the current guidelines used by national newborn screening in classifying variant galactosemia are recommended.

Key Words: galactosemia, Duarte variant, GALT genotype, newborn screening

Paper presented at the 11th Asia Pacific Conference on Human Genetics 2015 (APCHG 2015), September 16-19, 2015, Hanoi, Vietnam.

Corresponding author: Sylvia B. Capistrano-Estrada, MD

Institute of Human Genetics

National Institutes of Health

University of the Philippines Manila

623 Pedro Gil St., Ermita, Manila 1000 Philippines

Telephone: +6323070780

Email: scestrada@up.edu.ph
Introduction

Galactosemia is an inherited error in carbohydrate metabolism due to a deficiency in one of these enzymes: galactose-1-phosphate uridyltransferase (GALT), galactokinase (GALK), or UDP-galactose 4'-epimerase (GALE) (Figure 1). This then results in the non-breakdown of galactose causing cataracts, neurodevelopmental delay or liver dysfunction. Early manifestations may include failure to thrive and sepsis. Dietary galactose restriction throughout life is the treatment for individuals with GALT, GALK and generalized GALE deficiencies. All forms of galactosemia are inherited in an autosomal recessive manner. ${ }^{1}$

Breast milk/standard milk formula (lactose)
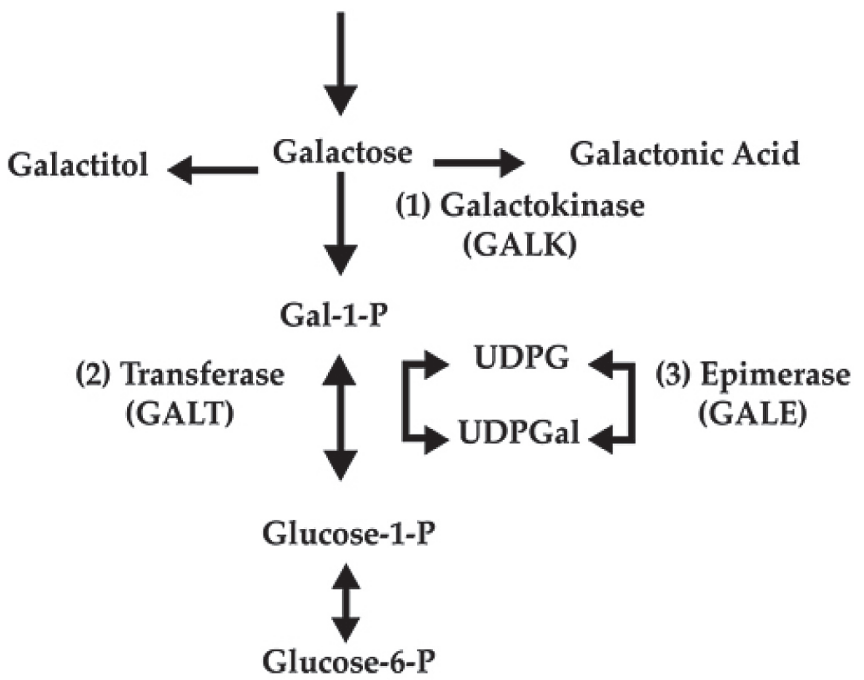

Figure 1. Metabolism of Galactose.

GALT deficiency (OMIM\# 230400), referred to as classic $(\mathrm{G} / \mathrm{G})$ galactosemia, is the most severe form of the disease, which can be life-threatening if left untreated..$^{2,3}$ This is caused by mutations in the GALT gene (Reference Assembly NC_000009.10) that have deleterious effect on the structure and function of the GALT enzyme and able to severely reduce or completely diminish enzyme activity. ${ }^{4}$ The $4-\mathrm{kb}$ GALT gene is located in chromosome 9p13 and contains 11 exons. ${ }^{5}$ There are over 360 reported GALT variations across the entire gene based on the GALT mutation database. ${ }^{6}$ 
Majority of these are classic mutations $(G)$ that cause severe or complete reduction of GALT enzyme activity. There are, however, mutations that only result to partial reduction of GALT activity. Some patients with variant galactosemia have been found to have residual GALT activity in the range of $1 \%$ to $5 \% .^{7}$ Typically, Duarte variant (D/G) galactosemia, is defined biochemically by a lower erythrocyte GALT enzyme activity of $14 \%$ to $25 \%$ of control and a genotype that shows one pathogenic GALT mutation in heterozygous state with Duarte allele. ${ }^{8}$ The partially impaired Duarte allele (D), also called Duarte-2 or D2, carry the p.N314D missense mutation in cis configuration with a 4-bp 5' deletion (c.-119_-116delGTCA) along with three intronic base changes [c.378-27G $>C$ or IVS4-27G $>C$, c.508$24 \mathrm{G}>\mathrm{A}$ or IVS5-24G $>\mathrm{A}$, and c.507+62G $>\mathrm{A}$ or IVS5+62G $>\mathrm{A}] .{ }^{9}$ In the newborn period, there are cases of $D / G$ mixed heterozygotes whose biochemical data mimic that of classic galactosemia patients resulting in a false positive newborn screening test. ${ }^{10}$ Molecular genetic testing has been used to confirm the diagnosis of classic galactosemia and to determine the presence of the Duarte allele.

The biochemical phenotype of children with Duarte galactosemia has been described by Ficicioglu et al. In their study, the red blood cell (RBC) galactose-1-phosphate (Gal1-P) concentrations were within the reference interval but the concentrations of other galactose metabolites, including RBC galactitol and RBC galactonate, were increased. The elevated concentrations of galactose metabolites other than the RBC Gal-1-P correlate with galactose intake. They stated that this observation denotes abnormal galactose burden. ${ }^{11}$

The observed irregularities in the biochemical profile of D/G galactosemics have led to the variability in patient management. The classification of Duarte variant as a benign condition and the implementation of treatment by dietary intervention are still subject to controversy. Various recommendations have been made including restriction of galactose in the diet during the first year of life and thereafter, a gradual introduction of this sugar while monitoring the clinical and biochemical response. Some physicians opt for non-treatment. 8,12

Individuals with variant galactosemia may be asymptomatic or manifest some mild aspects of classic galactosemia. 8,9 However, most previous studies report no clinical abnormalities despite biochemical abnormalities. Further, data on long-term outcomes among D/G galactosemics are limited. Ficicioglu et al. reported good clinical and developmental outcomes in D/G galactosemics during early childhood. ${ }^{12}$ Their research involved children aged 1-6 years old. Due to the normal clinical manifestations observed in their cohort, their group suggested the need for future studies that assess the cognitive and behavioral skills in older D/G individuals. ${ }^{12}$ On the other hand, Powell et al. reported that select developmental issues associated with special education, specifically involving speech and language, have been found among some children aged 3-10 years old with Duarte variant galactosemia. ${ }^{13}$ These neurodevelopmental outcomes were seen despite galactose restriction until one year of age, which is the treatment advocated by physicians. ${ }^{13}$ The seemingly contradicting results demonstrate that data on clinical and neurodevelopmental outcomes of variant galactosemia are still inconclusive.

The estimated prevalence of presumed variant galactosemia among Filipinos is 1:84,092 as detected by national newborn screening from 1996 to $2015 .{ }^{14}$ In screening for galactosemia among newborns in the Philippines, it has been observed that some neonates maintain normal blood galactose metabolites despite the absence of GALT enzyme activity. The newborns that exhibit these characteristics are presumed to have a variant form of galactosemia (pending genotyping). It must be noted, however, that this designation of "variant form galactosemia" is specific to the Philippine newborn screening program. Other newborn screening programs define variant galactosemia on the bases of lower GALT enzyme activity (14-25\%) and a genotype bearing at least one Duarte allele. The most common variant form of galactosemia is Duarte galactosemia.

Currently, there is no recommendation with regard to the long-term follow-up of variant galactosemia cases yet they continue to be detected through newborn screening. As a result of this, there can be follow-up concerns that may arise due to the non-existence of standardized guidelines on the treatment disposition of these patients. The uniform protocol of the local newborn screening centers is to follow these patients by monitoring their total blood galactose metabolites quarterly during the first year of life and yearly thereafter until five years (laboratory GMON Follow-up protocol). The families are counseled and instructed to keep these patients on unrestricted galactose containing diets and regular milk formula for the duration of the monitoring period. After the fifth year of monitoring, patients are discharged from the monitoring protocol if their galactose metabolites remain within normal and their GALT enzyme activity is recorded to be plus 1 or higher (estimated as $25 \%$ activity). Of the 108 patients monitored from 2002 to 2010, all maintained normal total galactose levels as of their last recorded data. It must be mentioned that while all patients were counseled to send in dried blood spots for galactose metabolite monitoring for 5 consecutive years, not all complied and hence, only twenty-six patients, whose galactose levels remained normal after five years were categorically discharged from the GMON follow-up protocol. Seventeen of the remaining 82 patients agreed to participate when recalled for physical and neurodevelopmental assessment in 2010. Five of the 17 were assessed to have developmental language delays in spite of normal total galactose levels. 
The main objectives of this study were to obtain the genotype information of the 17 patients who underwent physical and neurodevelopmental assessment, and describe the corresponding biochemical profile and clinical manifestations. From this pilot study, data may provide information for a better understanding of patients with presumed variant galactosemia as detected by the local newborn screening program, and may further serve as basis for long-term follow-up management. Molecular genetic testing would also confirm the diagnosis of the variant galactosemia patients.

\section{Methods}

This study was approved by the University of the Philippines Manila Research Ethics Board. Informed consent was obtained from the parent of each research participant prior to any study interview and procedures. Seventeen (17) patients were identified and contacted from the cohort of patients detected by newborn screening from 2002-2010, and who were monitored under the GMON protocol. These patients had existing records on physical, ophthalmologic and neurodevelopmental assessment before the start of the study. Biochemical data were obtained as part of the monitoring process. The biochemical criterion used by the newborn screening laboratory in classifying "variant galactosemia" is a qualitative estimate of the GALT enzyme activity of absent to trace (0-10\% activity) with normal total blood galactose metabolites. Upon recruitment, all study participants underwent repeat clinical, ophthalmologic and neurodevelopmental evaluations.
Genomic DNA was extracted from $4 \mathrm{ml}$ peripheral blood of the patients using the Qiagen QIAamp DNA Blood Midi Kit (Qiagen, Santa Clara, CA) based on the manufacturer's protocol. The 11 exons of the GALT gene with their flanking intronic regions were PCR-amplified in $10-\mu \mathrm{L}$ reaction volumes and visualized by agarose gel electrophoresis. Bidirectional sequencing of PCR products was performed using the Applied Biosystems 3130 Genetic Analyzer (Life Technologies, Carlsbad, CA). Mutations were identified by comparing the patient sample sequences with the published wild type GALT genomic DNA sequence (Reference Assembly NC_000009.10) using the Sequencher 4.10.1 software (Gene Codes Corporation, Ann Arbor, MI USA).

\section{Results and Discussion}

Thirteen of the eligible 17 patients with presumed variant galactosemia participated in the study. Three patients could not be reached; one patient declined. Among the 4 patients who did not participate, 2 were previously assessed to have developmental language delay. Of the 13 patients (ages 5 to 10 years and 10 months), 7 were females. All patients were ambulatory and well. Except for patient P3 who is menarchial, all were pre-pubertal. With respect to their physical growth, 11 had normal stature, 4 were overweight, and 1 was underweight. None of the patients exhibited cataracts nor hepatomegaly (Table 1 ).

None of the patients had the characteristic D/G galactosemia variant genotype (Table 2$)$. Five patients $(\mathrm{P} 1,3$, $4,6,8)$ were heterozygous for the classic galactosemia $(G)$ allele which may be a possible explanation for the low to

Table 1. Clinical and biochemical profile of patients with presumed variant galactosemia $(\mathrm{n}=13)$

\begin{tabular}{|c|c|c|c|c|c|c|c|}
\hline Patient & $\begin{array}{c}\text { Age at } \\
\text { Diagnosis } \\
\text { (days) }\end{array}$ & $\begin{array}{c}\text { Age at } \\
\text { Evaluation } \\
(2014)\end{array}$ & $\begin{array}{c}\text { Total Blood } \\
\text { Galactose (0-28 days) } \\
(\mathrm{mmol} / \mathrm{L})\end{array}$ & $\begin{array}{c}\text { GALT } \\
\text { Activity at } \\
0-28 \text { days }\end{array}$ & $\begin{array}{c}\text { Clinical and } \\
\text { Anthropometrics } \\
\text { (2014) }\end{array}$ & $\begin{array}{l}2^{\text {nd }} \text { Developmental } \\
\text { Assessment (2014) }\end{array}$ & $\begin{array}{l}1^{\text {st }} \text { Developmental } \\
\text { Assessment (2010) }\end{array}$ \\
\hline P1 & 3 & $6 \mathrm{yr}$ & 0.5 & Not detected & Normal & Normal & Normal \\
\hline P2 & 15 & 8 yr 5 mo & 0.5 & Not detected & $\begin{array}{l}\text { Tall } \\
\text { Overweight }\end{array}$ & Normal & Normal \\
\hline P3 & 27 & $10 y r 10$ mo & 0.2 & Not detected & Normal & Normal & Normal \\
\hline P4 & 3 & 5 yr 4 mo & 0.2 & Trace & $\begin{array}{l}\text { Tall } \\
\text { Overweight }\end{array}$ & Normal & $\begin{array}{l}\text { Expressive language } \\
\text { delay }\end{array}$ \\
\hline P5 & 33 & 5 yr 5 mo & 0.2 & Trace & $\begin{array}{l}\text { Short } \\
\text { Underweight }\end{array}$ & Global delay & Normal \\
\hline P6 & 11 & 5 yr 5 mo & 0.5 & Not detected & Normal & Normal & Normal \\
\hline P7 & 21 & 6 yr $1 \mathrm{mo}$ & 0.2 & Trace & $\begin{array}{l}\text { Tall } \\
\text { Normal weight; } \\
\text { Error of refrection }\end{array}$ & $\begin{array}{l}\text { Attention deficit } \\
\text { hyperactive disorder } \\
\text { (ADHD) }\end{array}$ & Normal \\
\hline P8 & 3 & 6 yr 3 mo & 0.2 & Trace & $\begin{array}{l}\text { Short } \\
\text { Normal weight } \\
\text { Error of refraction }\end{array}$ & Normal & Normal \\
\hline P9 & 2 & $5 \mathrm{yr}$ & 0.3 & Not detected & $\begin{array}{l}\text { Tall } \\
\text { Overweight }\end{array}$ & Normal & $\begin{array}{l}\text { Language delay } \\
\text { Mixed type }\end{array}$ \\
\hline P10 & 13 & 7 yr 2 mo & 0.6 & Not detected & $\begin{array}{l}\text { Tall } \\
\text { Overweight }\end{array}$ & Learning disability & Articulation problem \\
\hline P11 & 30 & 5 yr 10 mo & 0.2 & Not detected & $\begin{array}{l}\text { Tall } \\
\text { Normal weight }\end{array}$ & Normal & Normal \\
\hline P12 & 15 & $7 \mathrm{yr}$ & 0.7 & Not detected & Normal & Normal & Normal \\
\hline P13 & 15 & $7 \mathrm{yr}$ & 0.9 & Not detected & Normal & Normal & Normal \\
\hline
\end{tabular}


Table 2. Molecular profile of patients with presumed variant galactosemia $(n=13)$

\begin{tabular}{|c|c|c|c|c|c|}
\hline Patient & GALT Gene Mutations & Amino acid substitution & Genotype* $^{*}$ & References & Developmental 2014 \\
\hline P1 & c. $239 \mathrm{G}>\mathrm{A}$ & p.R80Q & $\mathrm{G} / \mathrm{N}$ & Novel & Normal \\
\hline P2 & c. [-119_-116delGTCA; 378-27G $\left.>C ; 508-24 \mathrm{G}>\mathrm{A} ; 940 \mathrm{G}>\mathrm{A}(;){ }^{*} 4 \mathrm{C}>\mathrm{T}^{\mathrm{a}}\right]$ & p.N314D & $\mathrm{D} / \mathrm{N}$ & $9,{ }^{a} 15$ & Normal \\
\hline P3 & c. $266 \mathrm{~A}>\mathrm{G}$ & p.Y89C & $\mathrm{G} / \mathrm{N}$ & Novel & Normal \\
\hline $\mathrm{P} 4$ & c. $502 \mathrm{G}>\mathrm{T}$ & p.V168L & $\mathrm{G} / \mathrm{N}$ & 6,16 & Normal \\
\hline P5 & None $^{* *}$ & None & $\mathrm{N} / \mathrm{N}$ & - & Global delay \\
\hline P6 & c. $239 \mathrm{G}>\mathrm{A}$ & p.R80Q & $\mathrm{G} / \mathrm{N}$ & Novel & Normal \\
\hline P7 & None ${ }^{* *}$ & None & $\mathrm{N} / \mathrm{N}$ & - & ADHD \\
\hline P8 & c. $239 \mathrm{G}>\mathrm{A}$ & p.R80Q & $\mathrm{G} / \mathrm{N}$ & Novel & Normal \\
\hline P9 & c.[-119_-116delGTCA; 378-27G>C; 508-24G >A; 940G>A(;) 687+62G>A'] & p.N314D & $\mathrm{D} / \mathrm{N}$ & 9, b15 & Normal \\
\hline P10 & None $^{* *}$ & None & $\mathrm{N} / \mathrm{N}$ & - & Learning Disability \\
\hline P11 & c.[-119_-116delGTCA; 378-27G>C; 508-24G>A; 940G>A(;) 820+36T>Cc] & p.N314D & $\mathrm{D} / \mathrm{N}$ & 9, cNovel & Normal \\
\hline P12 & None $^{* *}$ & None & $\mathrm{N} / \mathrm{N}$ & - & Normal \\
\hline P13 & None ${ }^{* *}$ & None & $\mathrm{N} / \mathrm{N}$ & - & Normal \\
\hline
\end{tabular}

${ }^{*} \mathrm{G}=$ classic allele, $\mathrm{D}=$ Duarte allele, $\mathrm{N}=$ normal allele

** for further molecular characterization

absent GALT activity. However, they were all clinically well and had no developmental concerns, consistent with normal total galactose metabolites. This further confirms that 2 pathogenic GALT alleles have to be present in order for galactose metabolites to elevate and cause clinically significant galactosemia. Three patients (P2, 9, and 11) were heterozygous for the Duarte allele, i.e., c.940G>A (p.N314D) in cis configuration with c.-119_-116delGTCA along with intronic base changes c.378-27G>C and c.508-24G>A. Additional intronic changes were also found with the Duarte allele, but it could not be determined whether these changes were on the same chromosome as the known Duarte mutations. Two of these are reported SNPs, c. ${ }^{*} 4 \mathrm{C}>\mathrm{T}$ (rs373627599) ${ }^{15}$ and c.687+62G>A (rs373173308) ${ }^{15}$, while one variation, c. $820+36 \mathrm{~T}>\mathrm{C}$, is unpublished and with unknown significance.

Three (3) missense mutations were detected: c.239G $>A$ (p.R80Q), c.266A>G (p.Y89C) and c.502G>T (p.V168L). The known pathogenic mutation p.V168L was also found in Filipino patients with classic galactosemia in our previous study. ${ }^{16}$ The p.R80Q and p.Y89C mutations are novel. In silico analyses through PolyPhen- $2^{17}$ and SIFT ${ }^{18}$ programs predict that these are both damaging mutations. The pathogenicity of the p.Y89C mutation is further supported by the existence of other disease-causing mutations at the same position (p.Y89H and p.Y89D) as reported in the GALT mutation database. ${ }^{6}$ The p.R80Q, p.Y89C, and p.V168L are classified as $\mathrm{G}$ alleles in Table 2. The carriers of p.Y89C and p.V168L had normal clinical findings. Of the 3 carriers of p.R80Q mutation, 1 was found to have an error of refraction, which may not be linked to its carrier status since the other 2 patients with the same genotype did not present with any clinical problems. These are novel mutations and literature has not reported patients with variant galactosemia having this genotype. Five patients ( $P$ $5,7,10,12,13)$ did not have detectable mutations in the coding region of the GALT gene and of these, 3 were each assessed to have one of the following: global delay (P5), attention deficit disorder (P7), and learning disability (P10). In addition, P7 also had an error of refraction. It is possible that these 5 patients do have the normal genotype $(\mathrm{N} / \mathrm{N})$, and that the problems presented by 3 of these patients may be coincidental and are unrelated to their presumed variant galactosemia condition. Of note is that patients 5 and $7 \mathrm{did}$ not have these learning delays during the previous neurodevelopmental assessment in 2010 (Table 1), hence, the strong argument for other factors possibly contributing to the current learning delays.

On the other hand, there is also a probability that they carry pathogenic GALT mutations that were not captured by the employed method. Therefore, additional molecular testing (e.g. sequence analysis of regulatory regions and whole gene deletion detection) may be needed to fully analyze the GALT gene in these patients. This may be especially warranted for P5, P7 and P10 who presented with some form of neurocognitive delay. In addition, P5 who had a dysplastic and small left ear, was referred for further diagnostic evaluation.

That none of the patients were confirmed to have Duarte variant $(D / G)$ galactosemia based on the results of molecular genetic testing strongly suggests that there may be a need to re-evaluate the algorithm including the cut-off values, method of determining galactose metabolites and GALT enzyme activity used by the local newborn screening program in classifying variant galactosemia patients. Our cohort was presumed to have variant galactosemia because of the absent or trace GALT enzyme activity measured by a qualitative method and inconsistently normal blood galactose metabolites. Nevertheless, our patients' genotypes, i.e. $\mathrm{G} / \mathrm{N}, \mathrm{D} / \mathrm{N}$, and $\mathrm{N} / \mathrm{N}$, are consistent with their normal total blood galactose levels but cannot explain the newborn screening result of "absent to trace" GALT activity. 
While we recognize the fact that the qualitative method to measure GALT enzyme activity has its limitations, it is what is readily available locally. Our findings put a strong reason to recommend molecular analysis for the confirmation of diagnosis of galactosemia and its variants.

\section{Conclusion and Recommendations}

Mutational analysis of the GALT gene revealed that none of the presumed variant galactosemia patients were Duarte variants. The observed genotypes reflect the normal total blood galactose levels in these patients. Both genotypes and galactose levels are inconsistent with the absent GALT enzyme activity. Therefore, our present cohort should be reclassified as not having Duarte variant galactosemia. Reevaluation and refinement of the method used by newborn screening in classifying variant galactosemia are, therefore, recommended.

Hypothesis testing and establishing of any genotypephenotype correlation were not performed because of the very small sample size. Similarly, there was no attempt to infer from the sample data what might be the characteristics of the whole population of Filipino patients with presumed variant galactosemia. For better profiling of this condition, we recommend molecular testing for the entire cohort for a more detailed molecular characterization. More studies may facilitate better approximation of long-term management and prognosis of variant galactosemia.

\section{Acknowledgment}

The authors thank the Section of Neurodevelopmental Pediatrics of the Philippine General Hospital for their assistance.

\section{Statement of Authorship}

All authors have approved the final version submitted.

\section{Author Disclosure}

All authors declared no conflict of interest.

\section{Funding Source}

This study is funded by the National Institutes of Health, University of the Philippines Manila and the Institute of Human Genetics.

\section{References}

1. Kaye CI. Newborn Screening Fact Sheets. Pediatrics. 2006; 118(3):e934-63.

2. Berry GT. Classic Galactosemia and Clinical Variant Galactosemia. 2000 Feb 4 [Updated 2014 Apr 3]. In: Pagon RA, Adam MP, Ardinger HH, et al., editors. GeneReviews ${ }^{\circledR}$ [Online]. Seattle (WA): University of Washington, Seattle; 1993-2017 [cited 2017 Mar]. Available from http://www.ncbi.nlm.nih.gov/books/NBK1518/.

3. Elsas LJ 2nd, Langley S, Paulk EM, Hjelm LN, Dembure PP. A molecular approach to galactosemia. Eur J Pediatr. 1995; 154(7 Suppl 2):S21-7.

4. Tang M, Facchiano A, Rachamadugu R, et al. Correlation assessment among clinical phenotypes, expression analysis and molecular modeling of 14 novel variations in the human galactose-1-phosphate uridyltransferase gene. Hum Mutat. 2012; 33(7):1107-15.

5. Leslie ND, Immerman EB, Flach JE, Florez M, Fridovich-Keil JL, Elsas LJ. The human galactose-1-phosphate uridyltransferase gene. Genomics. 1992; 14(2):474-80.

6. Calderon FR, Phansalkar AR, Crockett DK, Miller M, Mao R. Mutation database for the galactose-1-phosphate uridyltransferase (GALT) gene. Hum Mutat. 2007; 28(10):939-43.

7. Li Y, Ptolemy AS, Harmonay L, Kellogg M, Berry GT. Quantification of galactose-1-phosphate uridyltransferase enzyme activity by liquid chromatography-tandem mass spectrometry. Clin Chem. 2010; 56(5):772-80.

8. Fridovich-Keil JL, Gambello MJ, Singh RH, Sharer JD. Duarte Variant Galactosemia. 2014 Dec 4. In: Pagon RA, Adam MP, Ardinger HH, et al., editors. GeneReviews ${ }^{\circledR}$ [Online]. Seattle (WA): University of Washington, Seattle; 1993-2017 [cited 2017 Mar]. Available from https://www.ncbi.nlm.nih.gov/books/NBK258640/.

9. Carney AE, Sanders RD, Garza KR, et al. Origins, distribution and expression of the Duarte-2 (D2) allele of galactose-1-phosphate uridylyltransferase. Hum Mol Genet. 2009; 18(9):1624-32.

10. Dobrowolski SF, Banas RA, Suzow JG, Berkley M, Naylor EW. Analysis of common mutations in the galactose-1-phosphate uridyl transferase gene: new assays to increase the sensitivity and specificity of newborn screening for galactosemia. J Mol Diagn. 2003; 5(1):42-7.

11. Ficicioglu C, Hussa C, Gallagher PR, Thomas N, Yager C. Monitoring of biochemical status in children with duarte galactosemia: utility of galactose, galactitol, galactonate, and galactose 1-phosphate. Clin Chem. 2010; 56(7):1177-82.

12. Ficicioglu C, Thomas N, Yager C, et al. Duarte (DG) galactosemia: a pilot study of biochemical and neurodevelopmental assessment in children detected by newborn screening. Mol Genet Metab. 2008; 95(4):206-12.

13. Powell KK, Van Naarden Braun K, Singh RH, Shapira SK, Olney RS, Yeargin-Allsopp M. Long-term speech and language developmental issues among children with Duarte galactosemia. Genet Med. 2009; 11(12):874-9.

14. Padilla CD, Cutiongco-de la Paz EM. Genetics and genomic medicine in the Philippines. Mol Genet Genomic Med. 2016; 4(5):494-503.

15. National Center for Biotechnology Information. dbSNP: Short Genetic Variations [Online]. [cited 2017 Mar]. Available from https://www. ncbi.nlm.nih.gov/projects/SNP/.

16. Estrada SC, Canson DM, Silao CL. Mutational analysis of the GALT gene in Filipino patients. Kobe J Med Sci. 2013; 59(3):E106-111.

17. Adzhubei IA, Schmidt S, Peshkin L, et al. A method and server for predicting damaging missense mutations. Nat Methods. 2010; 7(4):248-9.

18. Kumar P, Henikoff S, Ng PC. Predicting the effects of coding nonsynonymous variants on protein function using the SIFT algorithm. Nat Protoc. 2009; 4(7):1073-81. 